\title{
Recent advances on potential drug therapies targeting COVID-19 and related coronaviruses
}

\author{
Shivraj Nile ${ }^{1}$, Arti Nile ${ }^{2}$, Shivkumar Jalde ${ }^{3}$, and Guoyin Kai ${ }^{1}$ \\ ${ }^{1}$ Zhejiang Chinese Medical University \\ ${ }^{2}$ Konkuk University \\ ${ }^{3}$ Jungwon University
}

November 17, 2020

\begin{abstract}
Coronaviruses (CoVs) are a large family of viruses responsible for the severe ill effects on human health. The most severe outbreak includes Severe Acute Respiratory Syndrome (SARS-CoV), Middle East Respiratory Syndrome (MERS-CoV) and Coronavirus disease 2019 (COVID-19) caused by Severe Acute Respiratory Syndrome coronavirus 2 (SARS-CoV-2). The COVID-19 poses major challenges to clinical management because no specific FDA-approved therapy yet to be available. Thus, the existing therapies are being used for the treatment of COVID-19, which are under clinical trials and compassionate use, based on in vitro and in silico studies. In this review, we summarized the potential therapies like small molecules, bioactive compounds, nucleoside and nucleotide analogs, peptides, antibodies, natural products, and synthetic compounds targeting the complex molecular interactions involved in COVID-19. In this review ¿230 natural and chemically synthesized drug therapies were described with their recent advances in research and development being done in terms of their chemical, structural and functional properties. Further, provided insights on possible targets for viral cells, viral proteins, viral replication, and different molecular pathways for the discovery of novel viral- and host-based therapeutic targets against SARS-CoV-2.
\end{abstract}

\section{Hosted file}

BJP-Manuscript.pdf available at https://authorea.com/users/376601/articles/493513-recentadvances-on-potential-drug-therapies-targeting-covid-19-and-related-coronaviruses 